\section{Kidney \\ Blood Pressure Research}

\title{
Serum Matrix Metalloproteinase-7 Level is Associated with Fibrosis and Renal Survival in Patients with IgA Nephropathy
}

\author{
Jian Zhang Pingping Ren Yucheng Wang Shi Feng Cuili Wang Xiujin Shen \\ Chunhua Weng Xiaobing Lang Zhiming Chen Hong Jiang Jianghua Chen
}

Kidney Disease Center, The First Affiliated Hospital, College of Medicine, Zhejiang University; Kidney Disease Immunology Laboratory, The Third Grade Laboratory, State Administration of Traditional Chinese Medicine of PR China; Key Laboratory of Multiple Organ Transplantation, Ministry of Health; Key Laboratory of Nephropathy, Zhejiang Province, China

\section{Key Words}

IgA nephropathy $\cdot$ Kidney disease progression - Matrix metalloproteinase-7 • Noninvasive • Renal fibrosis

\begin{abstract}
Background/Aims: In view of the latest findings that matrix metalloproteinase-7 (MMP7) acted as a vital marker and pathogenic mediator of renal fibrosis in a murine model, we hypothesized that serum MMP-7 level might serve as a noninvasive prognostic biomarker in IgA nephropathy (IgAN) patients. Methods: We conducted a retrospective follow-up study of 244 IgAN patients for a median of 81.9 months. Serum MMP-7 was detected at the time of diagnosis, and renal progression was assessed by Cox proportional hazards method. Results: Compared with healthy populations, the serum levels of MMP-7 were significantly elevated in IgAN patients. Besides, serum MMP-7 levels were well correlated with renal scarring lesions characterized by glomerular sclerosis and interstitial fibrosis. Follow-up analyses revealed that increased serum MMP-7 levels were linked with a greater risk of poor renal outcome with a hazard ratio of 1.898 per doubling MMP-7 concentration. By contrast with the first quartile, the risk of deterioration in renal function elevated such that the hazard ratio for the second quartile was $1.805,3.383$ for the third, and 5.173 for the fourth quartile of the MMP-7 level. Conclusions: This study showed that the higher serum MMP-7 levels were independently associated with renal fibrosis and poor prognosis in IgAN.
\end{abstract}




\section{Kidney Blood Pressure Research}

\section{Introduction}

IgA nephropathy (IgAN) is the most prevalent primary glomerular disease worldwide, characterized by predominant IgA deposition, either alone or with IgG, IgM, or both, in the glomerular mesangium $[1,2]$. Clinical manifestation and course of IgAN are quite heterogeneous, ranging from asymptomatic microscopic hematuria to rapidly progressive glomerulonephritis [3]. It estimates that approximately 30 - 50\% IgAN patients eventually reach end-stage renal disease (ESRD) within 20 years, obliging renal replacement therapy, dialysis or transplantation [1,4]. As a consequence, there exists enormous difficulty in choosing optimal treatments for different IgAN patients. Previous studies revealed that part of patients could benefit from immunosuppressive treatment [5-7]. Ideally, such therapy should only be given to patients who will finally progress to ESRD. Nevertheless, it remains unattainable to accurately predict the long-term clinical outcome for patients with IgAN as yet, especially in the early stages of disease [8].

Elevated serum creatinine concentration, minimal proteinuria, hyperuricemia and undesirable histological characteristics have been identified as baseline predictors of progression of IgAN [9-12]. Of them, histopathological examination of renal biopsy tissues is regarded as the gold standard for diagnosing IgAN, which manifests as an increase in mesangial matrix, endocapillary proliferation, along with focal necrosis and interstitial fibrosis [12]. Based on renal biopsy, the Oxford IgAN classification was formulated and represents a valuable prognostic tool for IgAN [13]. Interstitial fibrosis is one of the most important histological indicators, confirmed by a large number of original studies $[14,15]$. However, noninvasive markers of IgAN with prognostic value are still scarce, which will be of great significance for current clinical work.

Our previous results obtained in RNA-sequencing revealed that the expression of matrix metalloproteinase-7 (MMP-7) mRNA is markedly heightened in the kidney tissues of IgA patients [16]. MMP-7 is a secreted zinc- and calcium-dependent endopeptidase, which acts on a broad range of extracellular matrix components, such as type IV collagen, laminin, fibronectin, and entactin [17, 18]. Besides, activation of MMP-7 can function in the breakdown of extracellular matrix (ECM) and the cleavage of other substrates, Fas ligand, E-cadherin and also. The versatility of MMP-7 embodies itself a pivotal role in regulating a diverse range of cellular processes, including ECM remodeling and epithelial-mesenchymal transition(EMT) $[19,20]$. Not a few studies have reported that upregulation of MMP-7 was found in several fibrosis-related diseases, such as idiopathic pulmonary fibrosis [21], cirrhosis [22]. Moreover, recent publications demonstrated that the renal expression of MMP-7 was enhanced in kidney fibrosis as well, which correlates with $\beta$-catenin $[23,24]$.

Allow for previous reports of heightened renal MMP-7 expression in renal fibrosis and the significance of kidney fibrosis implicated in the pathogenesis of IgAN $[25,26]$, we hypothesized that the intrarenal fibrosis signature could be detected noninvasively by quantification of MMP-7 level in circulation and, it might serve as a noninvasive prognostic biomarker in IgAN. Since MMP-7 expression is little investigated in IgAN by now, in this study, we examined the association of serum MMP-7 level and histopathological change, as well as the renal progression of IgAN in a cohort of biopsy proven patients.

\section{Materials and Methods}

\section{Ethics Statement}

This study was performed in full adherence to the Declaration of Helsinki, and was approved by the Ethics Committee of the First Affiliated Hospital of College of Medicine of Zhejiang University. All patients and healthy controls provided written informed consent. Renal biopsies were operated according to our local regular clinical protocol. 


\section{Kidney Blood Pressure Research}

Patients and healthy controls

This was a single center retrospective study. Pathology Department electronic database from January 2003 to December 2012 was comprehensively reviewed to identify eligible IgAN patients with definite histological diagnosis. Patients with Henoch-Schonlein purpura, systemic lupus erythematosus, vasculitis were considered as unqualified. We further excluded patients aged $<18$ years or $>75$ years, and patients who initially presented with eGFR $<20 \mathrm{ml} / \mathrm{min}$ per $1.73 \mathrm{~m}^{2}$ were also eliminated in our study. In addition, subjects were excluded if they had missing data on baseline and short follow-up ( $<6$ months). Overall, a total of 244 IgAN patients were included in the present study. The healthy population controls were composed of 40 age and gender matched individuals with the IgAN patients. Among them, there were 19 males and 21 females, with their mean age was $37.5 \pm 8.3$ years. All healthy controls were at least 18 years old. In addition, $30 \mathrm{AKI}$ patients with reduced renal function not due to fibrotic conditions were also included as controls.

\section{Data Collection}

At the time of the renal biopsy, complete clinical data, including age, gender, systolic/diastolic blood pressure, serum creatinine, eGFR by CKD-EPI equation[27, 28], 24-h total protein excretion, macroscopic hematuria were recorded. Besides, history of preceding infection, medication use (renin angiotensin system blockade, corticosteroids, other immunosuppressive agents) as well as clinical and biological data were also collected.

\section{Histopathology}

Each specimen was routinely processed for light microscopy, immunofluorescence microscopy, and electron microscopy. Of them, Masson's trichrome staining was utilized to determine the fibrosis degree of the renal specimens. After the initiation of the present study, all available renal specimens were reassessed and graded by an experienced renal pathologist in a blind manner, in accordance with the Oxford classification[12]. The mesangial cellularity was scored 0 ( $<4$ mesangial cells per mesangial area), 1 ( $4-5$ cells), 2 ( 6 - 7 cells), and 3 ( $\geq 8$ cells) for each glomerulus. The mean score of all glomeruli was classified as M0 $(\leq 0.5)$ and M1 (>0.5). When more than half of glomeruli show score $\geq 1$, the case was classified as M1 without scoring each glomerulus. Segmental glomerulosclerosis and endocapillary hypercellularity were categorized as either present (S1 and E1) or absent ( $\mathrm{S} 0$ and E0). Tubular atrophy/ interstitial fibrosis was estimated semi-quantitatively and classified as T0 ( $0-25 \%$ of cortical area), T1 (26-50\% of cortical area), or $\mathrm{T} 2$ ( $\geq 50 \%$ of cortical area).

\section{Detection of serum MMP-7 level by ELISA}

Serum from IgAN patients, collected at the time of renal biopsy, and from controls were aliquoted and stored at $-80^{\circ} \mathrm{C}$ until the time of assays. Serum MMP-7 level was measured using a commercial human MMP-7 enzyme-linked immunosorbent (ELISA) kit, according to manufactory's instructions (DMP700, R\&D System, USA). All samples were tested in duplicate with five-fold dilutions. The results for MMP-7 were expressed as $\mathrm{ng} / \mathrm{ml}$.

\section{Study Outcome}

The composite end point of the present study was defined by the onset of ESRD and a 50\% decline in eGFR from the time of diagnosis of IgAN. ESRD was defined as the requirement for renal replacement therapy or transplantation.

\section{Statistical analyses}

Above all, original MMP-7 levels were highly skewed, so that $\log _{2}$-transformation was used. Continuous variables were reported as means and standard deviations, or median and IQR. Qualitative variables were summarized by ratios and percentages. Differences in means for continuous variables were judged using unpaired Student's t-test or non parametric test or analysis of variance, and differences in proportions were calculated by $\chi^{2}$ test. Correlations between $\log _{2}$ MMP-7 and biological data and MEST scores were calculated by the Pearson and Spearman correlation coefficient. Besides, univariate and multivariate Cox regression analyses were conducted. Serum MMP-7 was first analyzed as a continuous variable with HRs calculated per doubling original MMP-7 concentrations, and then the MMP-7 quartile as a categorical variable, with the lowest quartile regarded as the reference group. 


\section{Kidney \\ Blood Pressure Research}

Table 1. Baseline demographic, clinical, biochemical data and levels of serum MMP-7 in 244 patients with IgAN

\begin{tabular}{|c|c|c|c|}
\hline Characteristics & \multicolumn{3}{|c|}{ Mean \pm s.d. (range or percentage) } \\
\hline Age (year) & \multicolumn{3}{|c|}{$38.95 \pm 12.4(18-73)$} \\
\hline Gender (male) & \multicolumn{3}{|l|}{$117(48 \%)$} \\
\hline Hypertension (\%)a & \multicolumn{3}{|l|}{$140 / 244(57.4 \%)$} \\
\hline Diabetes (\%) & \multicolumn{3}{|l|}{$13(5.3 \%)$} \\
\hline Gross hematuria & \multicolumn{3}{|l|}{$22.1 \%$} \\
\hline Initial proteinuria (g/24h) & \multicolumn{3}{|l|}{$2.52 \pm 1.98$} \\
\hline$<0.3(\%)$ & \multicolumn{3}{|l|}{$8 / 244(3.3 \%)$} \\
\hline $0.3-0.99(\%)$ & \multicolumn{3}{|l|}{$40 / 244(16.4 \%)$} \\
\hline $1.0-2.99(\%)$ & \multicolumn{3}{|l|}{$114 / 244(46.7 \%)$} \\
\hline$\geq 3.0(\%)$ & \multicolumn{3}{|l|}{$82 / 244(33.6 \%)$} \\
\hline eGFR $\left(\mathrm{ml} / \mathrm{min}\right.$ per $\left.1.73 \mathrm{~m}^{2}\right)$ & \multicolumn{3}{|l|}{$78.64 \pm 36.3$} \\
\hline Stage $1,2,3$ and 4 CKD (KDOQI) $)^{b}$ & \multicolumn{3}{|c|}{110 (45.1\%), 43 (17.6\%), 61 (25\%), 30 (12.3\%) } \\
\hline Follow-up interval (month, median, IQR) & \multicolumn{3}{|c|}{$81.9(46.3-116.6)$} \\
\hline Serum MMP-7 (ng/ml, median, IQR) & \multirow{2}{*}{\multicolumn{3}{|c|}{$5.99(3.53-10.18)$}} \\
\hline Therapy (\%) & & & \\
\hline ACE inhibitors or ARBs & \multicolumn{3}{|l|}{$80 \%$} \\
\hline steroid & \multicolumn{3}{|l|}{$35.7 \%$} \\
\hline $\begin{array}{l}\text { Any other immunosuppressive agents } \\
\text { (cyclophosphamide, MMF, or others) }\end{array}$ & \multirow{2}{*}{\multicolumn{3}{|c|}{$31.6 \%$}} \\
\hline Histological grading $(\%)^{c}$ & & & \\
\hline M item & M0 n = $107(43.9 \%)$ & $M 1 \mathrm{n}=137(56.1 \%)$ & \\
\hline E item & E0 n = $200(82 \%)$ & $\mathrm{E} 1 \mathrm{n}=44(18 \%)$ & \\
\hline S item & $\mathrm{S} 0 \mathrm{n}=100(41 \%)$ & $\mathrm{S} 1 \mathrm{n}=144(59 \%)$ & \\
\hline T item & $\mathrm{T} 0 \mathrm{n}=161(66 \%)$ & $\mathrm{T} 1 \mathrm{n}=60(24.6 \%)$ & $\mathrm{T} 2 \mathrm{n}=23(9.4 \%)$ \\
\hline Crescent & $\begin{array}{c}\text { Absent } \\
\mathrm{n}=172(70.5 \%)\end{array}$ & $\begin{array}{c}\text { Present } \\
\mathrm{n}=72(29.5 \%)\end{array}$ & \\
\hline
\end{tabular}

Abbreviation: ACE, angiotensin-converting enzyme; ARB, angiotensin II receptor blocker; eGFR, estimated Glomerular Filtration Rate by CKD-EPI equation; IQR, interquartile range; MMF, mycophenolate mofetil; IgAN, IgA nephropathy. M1, mesangial hypercellularity > 0.5; E1, presence of endocapillary hypercellularity; S1, presence of segmental glomerulosclerosis; $\mathrm{T} 1$, tubular atrophy/interstitial fibrosis $26-50 \%$ of cortical area; T2, tubular atrophy/interstitial fibrosis $>50 \%$ of cortical area. aHypertension was defined as SBP of greater than $140 \mathrm{~mm} \mathrm{Hg}$, and/or DBP of greater than $90 \mathrm{~mm} \mathrm{Hg}$ at resting, or use of anti-hypertension medication. bCKD stage 1, 2, 3, and 4 were divided by eGFR $\geq 90,60-89,30-59$, and $15-29$, respectively. ${ }^{c}$ Histological grading was classified according to the Oxford IgAN classification.

Finally, Harrell's C index was calculated to compare the different discriminatory ability between Cox regression models. Using this index, we addressed the power of serum MMP-7 level in the prediction of renal survival. A two-sided P-value $<0.05$ was considered statistically significant. All statistical analyses were performed using SPSS package, version 22.0 (Chicago, Illinois, USA) and STATA version 13.0 software (STATA Corp, College Station, Texas, USA).

\section{Results}

Baseline characteristics of IgA patients

Patient demographic, clinical and biochemical data at the time of biopsy for IgAN are shown in Table 1 . Among the 244 IgAN patients, there were 117 males and 127 females, with mean was $38.95 \pm 12.4$ years. At diagnosis, $140(57.4 \%)$ patients presented with hypertension, $54(22.1 \%)$ had gross hematuria. Initial average proteinuria level was $2.52 \pm$ 1.98 per $24 \mathrm{~h}$ and average eGFR was $78.63 \pm 36.3 \mathrm{ml} / \mathrm{min}$ per $1.73 \mathrm{~m}^{2}$. The median follow-up time was 81.9 (46.3 - 116.6) months. 


\section{Kidney Blood Pressure Research}

Kidney Blood Press Res 2017;42:541-552

\begin{tabular}{l|l}
\hline DOI: 10.1159/000477132 & ( 2017 The Author(s). Published by S. Karger AG, Basel
\end{tabular} Published online: September 18, 2017 www.karger.com/kbr

Zhang et al.: Serum MMP-7 and Renal Survival in IgAN
Besides, detailed information for $30 \mathrm{AKI}$ patients with reduced renal function was displayed in Table 2.

\section{Pathological features}

Histological characteristics in respect to $M, E, S, T$ items were also shown in Table 1 . Among all biopsy specimens, 161(66\%), 60 (24.6\%), 23 (9.4\%) were graded T0, T1, T2, respectively. 100 (41\%), 144(59\%) were scored S0, S1. Additionally, crescents were found in $72(29.5 \%)$ cases.
Table 2. Baseline demographic, clinical, and biochemical data in 40 healthy controls and 30 AKI patients

\begin{tabular}{|c|c|c|}
\hline Variable & $\begin{array}{l}\text { Healthy controls } \\
\qquad(\mathrm{n}=40)\end{array}$ & $\begin{array}{c}\text { AKI patients } \\
(\mathrm{n}=30)\end{array}$ \\
\hline Age (years) & $37.5 \pm 8.3$ & $47.3 \pm 12.4$ \\
\hline Gender (male) & $19(47.5 \%)$ & $15(50 \%)$ \\
\hline sBP (mmHg) & $120.5 \pm 6.2$ & $115.6 \pm 8.3$ \\
\hline $\mathrm{dBP}(\mathrm{mmHg})$ & $73.7 \pm 3.6$ & $65.3 \pm 5.8$ \\
\hline Diabetes (n) & 0 & 3 \\
\hline Serum albumin (g/L) & $37.4 \pm 3.6$ & $32.8 \pm 7.2$ \\
\hline Proteinuria levels (g/24h) & N.A. & N.A. \\
\hline eGFR $\left(\mathrm{ml} / \mathrm{min}\right.$ per $\left.1.73 \mathrm{~m}^{2}\right)$ & $112.4 \pm 8.3$ & $63.3 \pm 16.5$ \\
\hline \multicolumn{3}{|c|}{$\begin{array}{l}\text { Data are expressed as mean } \pm \text { SD. Abbreviation: eGFR, estimated } \\
\text { Glomerular Filtration Rate by CKD-EPI equation. N.A., not } \\
\text { available. }\end{array}$} \\
\hline
\end{tabular}

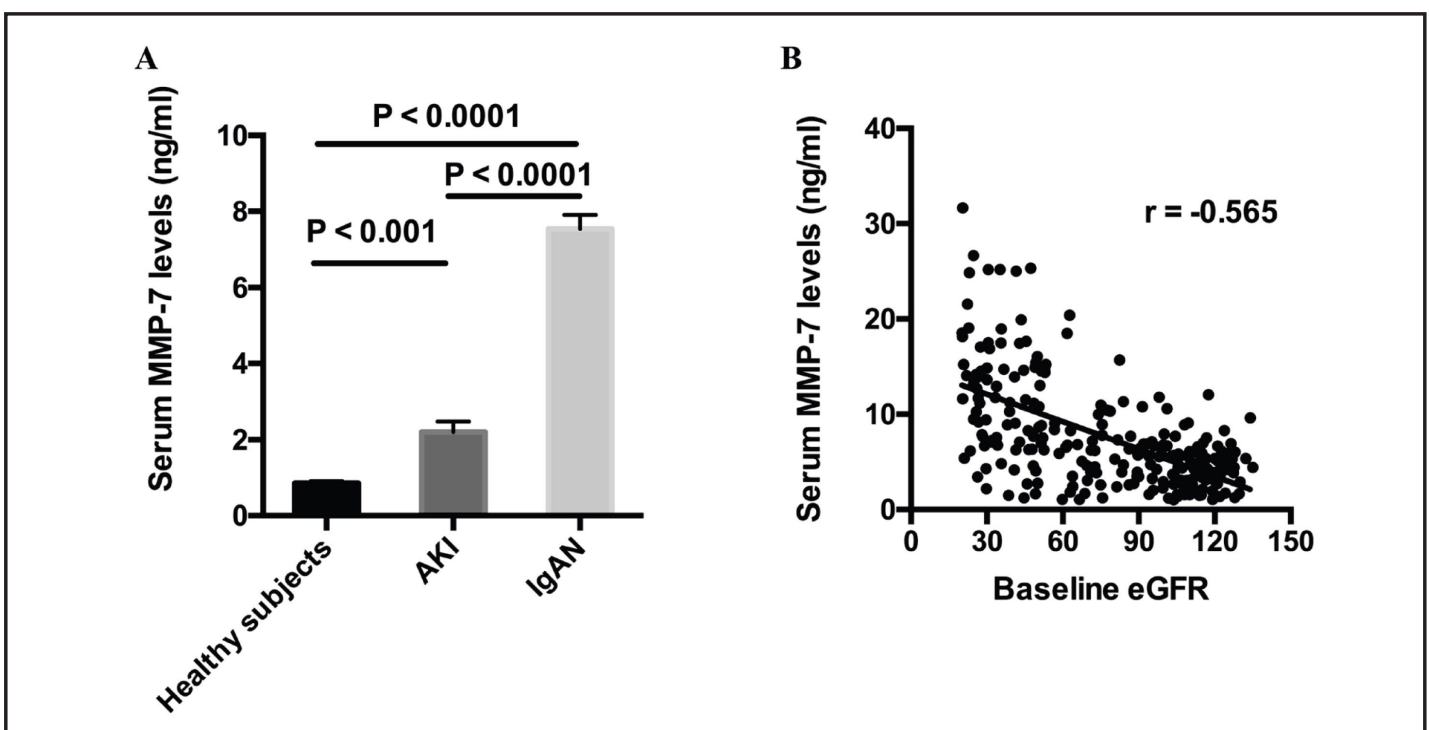

Fig. 1. Serum MMP-7 levels are elevated in IgAN patients. (A) Serum MMP-7 levels in patients with IgAN (n = 244), healthy control $(n=40)$ and 30 AKI patients. (B) Serum MMP-7 levels were negatively related with kidney function of IgAN patients.

Serum MMP-7 levels are increased in IgAN patients

Previous study suggested that MMP-7 was detectable in the urine of animal models of CKD [24]. Here we firstly compared serum MMP-7 concentrations in 244 IgAN patients with 40 healthy controls. Results revealed that serum MMP-7 levels were notably increased in patients with IgAN (median: $5.99 \mathrm{ng} / \mathrm{ml}$, interquartile range (IQR) $3.53-10.18 \mathrm{ng} / \mathrm{ml}, \mathrm{P}$ $<0.00001$ ), as presented in Figure 1A. Besides, elevation of serum MMP-7 levels was also found in $30 \mathrm{AKI}$ patients (median: $1.84 \mathrm{ng} / \mathrm{ml}$, IQR $1.06-2.66 \mathrm{ng} / \mathrm{ml}, \mathrm{P}<0.001$ ). However, the magnitude of increase was significantly smaller than that in patients with IgAN (Figure 1A). Then serum MMP-7 levels were further compared between AKI patients and a subset of IgAN patents with a comparable eGFR. As expected, serum MMP-7 levels were still much higher in the IgAN patients with similar renal function (median: 5.89, IQR $3.48-7.19 \mathrm{ng} /$ $\mathrm{ml}, \mathrm{P}<0.0001$ ). At last, we found that the levels of serum MMP-7 were closely related to the severity of kidney function decline among IgAN patients, with correlation coefficient $r=$ -0.565 (Figure 1B). 


\section{Kidney Blood Pressure Research}

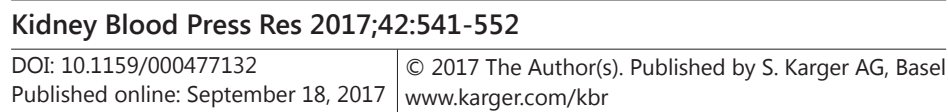

Table 3. Baseline data of clinical and pathological features and levels of serum MMP-7 of four groups defined by quartiles of serum MMP-7 in IgAN partients

\begin{tabular}{|c|c|c|c|c|c|}
\hline \multirow[t]{2}{*}{ Groups } & 1 & 2 & 3 & 4 & \\
\hline & $\begin{array}{c}\mathrm{ng} / \mathrm{ml} \\
\text { median (IQR) }\end{array}$ & $\begin{array}{c}\mathrm{ng} / \mathrm{ml} \\
\text { median (IQR) }\end{array}$ & $\begin{array}{c}\mathrm{ng} / \mathrm{ml} \\
\text { median (IQR) }\end{array}$ & $\begin{array}{c}\mathrm{ng} / \mathrm{ml} \\
\text { median (IQR) }\end{array}$ & $P$ value \\
\hline Group & $\begin{array}{c}2.11 \\
(1.55-2.90)\end{array}$ & $\begin{array}{c}4.73 \\
(4.16-5.54)\end{array}$ & $\begin{array}{c}7.12 \\
(6.61-8.28)\end{array}$ & $\begin{array}{c}14.62 \\
(11.73-17.91)\end{array}$ & \\
\hline Number & 61 & 61 & 61 & 61 & \\
\hline Gender (male) & $30 / 61$ & $22 / 61$ & $31 / 61$ & $34 / 61$ & 0.16 \\
\hline Age (year) & $37.39 \pm 11.88$ & $37.79 \pm 10.47$ & $40.33 \pm 13.94$ & $40.30 \pm 13.05$ & 0.616 \\
\hline Hypertension (\%) & $30(49.2 \%)$ & $29(47.5 \%)$ & $40(65.6 \%)$ & $41(67.2 \%)$ & 0.042 \\
\hline Diabetes $(\mathrm{n})$ & 2 & 5 & 3 & 3 & 0.746 \\
\hline Proteinuria $(\% \geq 1 \mathrm{~g} / 24 \mathrm{~h})$ & $41(67.2 \%)$ & $44(72.1 \%)$ & $53(86.9 \%)$ & $58(95.1 \%)$ & $<0.001$ \\
\hline Gross hematuria (\%) & $10(16.4 \%)$ & $19(31 \%)$ & $12(19.7 \%)$ & $13(21.3 \%)$ & 0.233 \\
\hline $\begin{array}{l}\text { eGFR }\left(\mathrm{ml} / \mathrm{min} \text { per } 1.73 \mathrm{~m}^{2}\right)^{*} \\
\text { Treatment }(\%)\end{array}$ & $41,12,6,2$ & $43,11,5,2$ & $22,13,19,7$ & $4,7,31,19$ & $<0.001$ \\
\hline Steroid & $18(29.5 \%)$ & $17(27.9 \%)$ & $25(41 \%)$ & $27(44.3 \%)$ & 0.148 \\
\hline $\begin{array}{l}\text { Any other } \\
\text { immunosuppressive agents }\end{array}$ & $14(23 \%)$ & $17(27.9 \%)$ & $22(36.1 \%)$ & $24(39.3 \%)$ & 0.19 \\
\hline $\begin{array}{l}\text { ACE inhibitors or ARBs } \\
\text { Oxford classification (\%) }\end{array}$ & $45(73.8 \%)$ & $48(78.7 \%)$ & $50(82 \%)$ & $52(85.2 \%)$ & 0.435 \\
\hline M1 & $27(44.3 \%)$ & $28(45.9 \%)$ & $35(57.4 \%)$ & 47 (77\%) & 0.001 \\
\hline E1 & $12(19.7 \%)$ & $6(9.8 \%)$ & $7(11.5 \%)$ & $19(31.1 \%)$ & 0.008 \\
\hline S1 & $32(52.5 \%)$ & $32(52.5 \%)$ & 36 (59\%) & $44(72.1 \%)$ & 0.089 \\
\hline $\mathrm{T} 1$ & $4(6.6 \%)$ & $6(9.8 \%)$ & $22(36.1 \%)$ & $28(45.9 \%)$ & $<0.001$ \\
\hline $\mathrm{T} 2$ & 0 & 0 & $4(6.6 \%)$ & $19(31.1 \%)$ & $<0.001$ \\
\hline End point & $6(9.8 \%)$ & $10(16.4 \%)$ & $29(47.5 \%)$ & $47(77 \%)$ & $<0.001$ \\
\hline
\end{tabular}

IgAN patients were next divided into four equal groups according to the quartiles of the MMP-7 distribution; group 1: serum MMP-7 levels < $3.54 \mathrm{ng} / \mathrm{ml}$; group 2: serum MMP-7 between 3.54 and $5.98 \mathrm{ng} / \mathrm{ml}$; group 3: serum MMP-7 levels between 5.98 and $9.99 \mathrm{ng} / \mathrm{ml}$; and group 4, serum MMP-7 levels $>10.24 \mathrm{ng} / \mathrm{ml}$. No significant association between MMP7 levels and age, gender, gross hematuria and treatment was observed (Table 3). However, there existed a distinct trend for lower eGFR, higher proteinuria, increased proportions of hypertension and more severe histological changes in groups with higher MMP-7 levels.

Association of serum MMP-7 levels with MEST scores.

Considering that MMP-7 as a direct target of Wnt/ $\beta$-catenin, involved in renal fibrogenesis [24], we explored the possible association between serum MMP-7 levels and MEST scores in IgAN patients. Results showed that except E item, M, S, T items were all strongly linked with $\log _{2}$ MMP-7, with $r=0.29,0.14,0.57$, respectively. In other words, serum MMP-7 levels were significantly raised in patients with interstitial $(\mathrm{T})$, glomerular $(\mathrm{S})$ fibrotic lesions and mesangial hypercellularity (M), especially in T2 patients (Figure 2).

Prognostic value of serum MMP-7 level for renal outcome in IgAN patients

During a median follow-up of $81.9(46.3$ - 116.6) months, $92(37.7 \%)$ patients reached the composite end point, comprising ESRD $(n=64)$ and 50\% decline in eGFR ( $n=28)$. Baseline clinical and pathological variables were analyzed for associations with renal outcome using Cox proportional hazards models.

Unadjusted and multivariable adjusted hazard ratios (HRs) are shown in Table 4, according to baseline MMP-7 levels, expressed as a continuous variable or in quartiles. In ad- 


\section{Kidney \\ Blood Pressure Research}

justed analysis of model 1including sex, age, and baseline clinical variables (eGFR, proteinuria, hypertension), higher MMP-7 levels were independently related with adverse renal outcome (HR, per doubling MMP7 1.948; 95\% confidence interval (CI), 1.478 - 2.567, $P=0.000002$ ). Further adjustment for history of preceding infection, gross hematuria and steroid use, $\log _{2}$ MMP-7 remained notably associated with renal function decline (HR $=2.023,95 \%$ CI 1.478 - 2.567, P = 0.000001). Considering the relevance between serum MMP-7 levels and histological variables in IgAN patients, the components
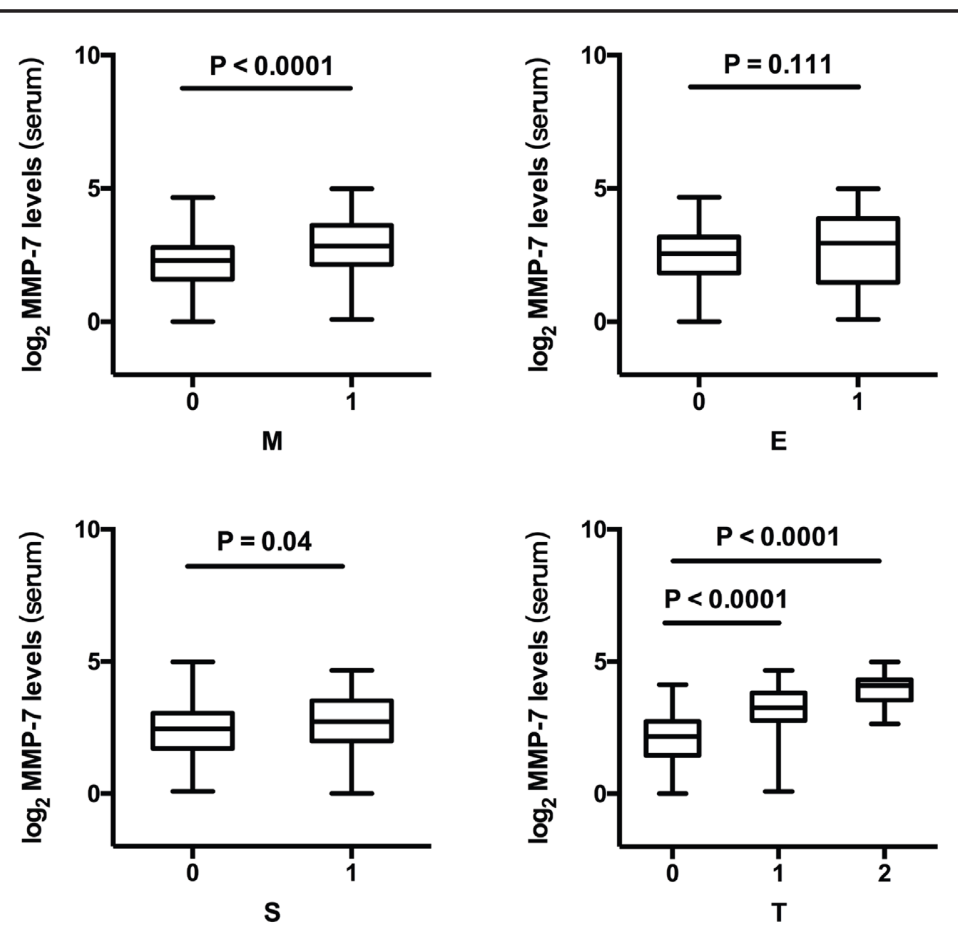

Fig. 2. Serum MMP-7 levels are associated with mesangium proliferation (M), segmental glomerulosclerosis (S) and interstitial fibrosis (T). Serum MMP-7 levels were presented as $\log _{2}$-transformation.

Table 4. Risks of composite end-point $\log _{2}$-transformed MMP-7 and ascending quartiles

\begin{tabular}{|c|c|c|c|c|c|}
\hline & & & Hazard ratio & $5 \%$ confidence interva & and P-value) \\
\hline & $\begin{array}{l}\text { MMP-7, median } \\
\text { (range), ng/ml }\end{array}$ & Unadjusted & Model 1 & Model 2 & Model 3 \\
\hline & posite end point & er doubling & & & \\
\hline & & $2.811(2.226,3.549)$ & $1.948(1.478,2.567)$ & $2.023(1.524,2.686)$ & $1.898(1.408,2.559)$ \\
\hline & & $3.7447 \mathrm{e}-18$ & 0.000002 & 0.000001 & 0.000026 \\
\hline & -7 quartiles & & & & \\
\hline 1 & $\begin{array}{c}2.11 \\
(100,3.53)\end{array}$ & 1 (reference) & 1 (reference) & 1 (reference) & 1 (reference) \\
\hline 2 & 4.73 & $1.727(0.627,4.754)$ & $1.816(0.656,5.022)$ & $1.691(0.61,4.688)$ & $1.805(0.649,5.021)$ \\
\hline & $(3.54,5.98)$ & 0.29 & 0.25 & 0.312 & 0.258 \\
\hline 3 & 7.13 & $5.791(2.403,13.958)$ & $3.569(1.454,8.761)$ & $3.616(1.462,8.942)$ & $3.383(1.35,8.481)$ \\
\hline & $(6.01,9.99)$ & 0.000091 & 0.005 & 0.005 & 0.009 \\
\hline 4 & 14.62 & 14.182 & 5.873 & 5.931 & 5.173 \\
\hline & $(10.25,31.66)$ & $(6.050,33.246)$ & $(2.376,14.518)$ & $(2.381,14.776)$ & $(2.023,13.225)$ \\
\hline & & $1.0543 \mathrm{e}-9$ & 0.000126 & 0.000132 & 0.001 \\
\hline
\end{tabular}

Abbreviations: $\log _{2}$ MMP-7, $\log _{2}$-transformed MMP-7. ${ }^{\mathrm{a}}$ Model 1 adjusted for sex, age, and baseline clinical variables (estimate glomerular filtration rate (eGFR), proteinuria, and hypertension (yes or no)). Hypertension (yes or no) was analyzed as dichotomous data. ${ }^{b}$ Model 2 adjusted for covariates in model 1 plus history of preceding infection, gross hematuria and steroid use (yes or no). Three variables were all analyzed as dichotomous data. ${ }^{C}$ Model 3 adjusted for covariates in model 2 plus M, E, S, T scores. Composite end point was defined as 50\% decline of eGFR ( $n=28)$, end-stage renal disease $(n=64)$. Unadjusted model analyzed MMP-7 as continuous data.

of the Oxford-MEST lesion classification were additionally adjusted in the model 3. Finally, results demonstrated that serum MMP-7 could still significantly predict the risk of kidney failure $(\mathrm{HR}=1.898,95 \mathrm{CI} \% 1.408-2.559, \mathrm{P}=0.000026)$. 


\section{Kidney Blood Pressure Research}

For a more accurate understanding of the prognostic value of MMP7 , quartiles of MMP-7 were used in our regression models. Compared with the first quartile of MMP-7, regarded as reference group, the risks of renal failure were strikingly elevated by the quartile of MMP-7 levels, especially in the third and fourth group, the HRs were 3.383, 95\% CI $(1.35,8.481)$ and $5.173,95 \%$ CI $(2.023,13.225)$, respectively. Nevertheless, no distinct increase was noted in the second group (HR $=1.805$, $95 \%$ CI $0.649-5.021, \mathrm{P}=0.258$ ).

The Oxford classification of IgAN does not account for glomerular crescents. A recent large IgAN cohorts revealed that crescents predicted a higher risk of $50 \%$ decline in eGFR or ESRD in patients without receiving immunosuppression [29]. Moreover, having crescents in at least one fourth of glomeruli independently related with a combined event in patients, no matter receiving immunosuppression or not. Hence, presence of crescents or not was further added into the fully adjusted model. Additional adjustment for crescents did not attenuate the association between serum MMP7 and poor renal outcome (HR, per doubling MMP-7 1.87, 95\% CI 1.383 - 2.528, $\mathrm{P}=0.000047$ ).

As depicted in Figure 3, renal outcome dramatically deteriorated with the quartiles of serum MMP-7 levels. Of IgAN patients with the higher levels of serum MMP-7, cumulative renal survival was significantly lower $(\mathrm{P}<0.001)$.

Comparative prognostic values of serum MMP-7 level and Oxford MEST for prediction of renal outcome

At last, we calculated Harrell's C index in multivariate Cox regression models to compare the predictive capacity of serum MMP level with Oxford-MEST scores (Table 5). Oxford-MEST lesions are well-known risk factors for renal progression, with a high C-statistic in model 1 (C-statistic, 0.819, 95\% CI: 0.779 - 0.859). Of note, model 2 in which MMP-7 was included yielded a similar C-statistic (C-statistic, 0.829, 95\% CI; $0.791-0.866$ ), P $=0.139$.

\section{Discussion}

The present study demonstrated that serum MMP-7 levels were remarkably associated with deterioration of renal outcome in patients with IgAN, independent of baseline eGFR, proteinuria, and unsatisfied histopathological features, such as glomerular sclerosis, tubule-
Table 5. C-statistics for prediction of renal outcome of end-stage renal disease and 50\% decline in eGFR using multivariate Cox's regression models

\begin{tabular}{lcc}
\hline Model & C-statistics $(95 \% \mathrm{CI})$ & $\begin{array}{l}\mathrm{P} \text { for difference of } \\
\text { C-statistics compared } \\
\text { with models }\end{array}$ \\
\hline Model 1 $^{\mathrm{a}}$ & $0.819(0.779-0.859)$ & \\
Model 2 $^{\mathrm{b}}$ & $0.829(0.791-0.866)$ & 0.139 \\
\hline
\end{tabular}

Abbreviation: eGFR: estimated glomerular filtration; CI, confidence interval. aModel 1: baseline clinical variables + (used as continuous variable).

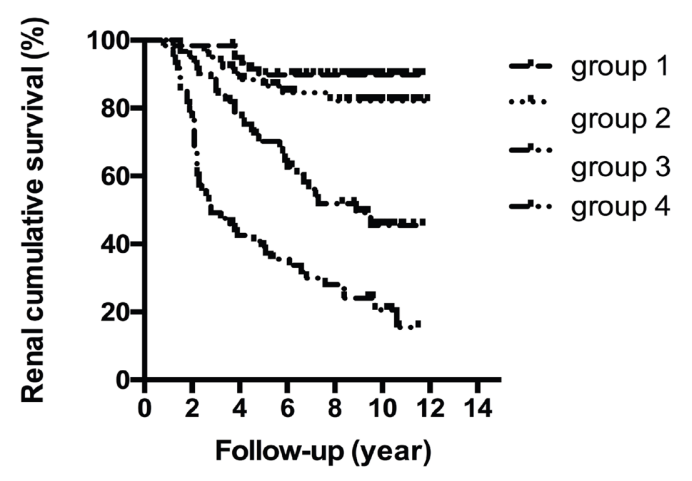

Fig. 3. Kaplan-Meier analysis of cumulative renal survival in IgAN patients with four quartiles serum MMP-7 levels. The renal outcome deteriorated by the quartile of serum MMP7 level (log-rank test, $\mathrm{P}<0.0001$ ). The time zero was renal biopsy. The division among the four groups was based on quartile of MMP-7 level expressed as 3.54, 5.98, $10.24 \mathrm{ng} / \mathrm{ml}$. 


\section{Kidney Blood Pressure Research}

interstitial fibrosis, or even crescents. Moreover, compared with the model using the OxfordMEST scores, serum MMP-7 displayed a comparable predictive power for progression of ESRD.

A highly variable course was observed among different IgAN patients. While a subset of patients are under a rather favorable disease trajectory, some patients manifest with rapid decline in renal function. Therefore, it is difficult to predict long-term outcomes between individuals. Hypertension, renal insufficiency and heavy proteinuria are known risk factors for poor prognosis in the context of IgAN [30]. Nevertheless, identification of other putative factors implicated in the progression of IgAN is still a developing area of interest.

Interstitial fibrosis and glomerular sclerosis are two potent prognostic factors for developing ESRD in patients with IgAN [14]. As the final common pathway of almost all progressive chronic kidney diseases, renal fibrosis may already be present before a decline in renal function is detectable by other clinical methods. However, early detecting kidney fibrosis has to rely on an invasive test of renal biopsy, which may be unnecessary for the majority of IgAN patients.

Recently, mounting studies were devoting to identifying possible fibrosis-related biomarkers with prognostic values for IgAN patients. Protein and/or mRNA biomarkers in urine and peripheral blood are most widely investigated. One latest cohorts study compared 92 IgAN patients with 53 healthy controls, results revealed that a combination of markers of collagen type III turnover, in particular the urinary Pro-C3/C3M ratio, was a promising novel, non-invasive diagnostic and prognostic tool to reflect disease severity and progression in patients with IgAN [31].

MiRNAs, regarded as new biomarkers and participators, were also identified in the physio-pathological processes of renal fibrosis. After measuring renal expression of miR21-5p, miR-214-3p, and miR-199a-5p levels, Hennino et al. confirmed that miR-21-5p acted as a "fibromiRs", participated in the course of IgAN and closely linked with renal survival [25]. Furthermore, several publications suggested that circulating miRNAs levels might also represent a novel, predictive and reliable blood marker of kidney fibrosis [32-34].

Both in previous vivo and vitro studies have firmly established that MMP-7 played a pivotal role in the development of kidney fibrosis. In the murine models of obstructive nephropathy and focal segmental glomerulosclerosis, He et al. [24] observed that the pattern and extent of MMP-7 expression were positively associated with Wnt/ $\beta$-catenin signaling. What is more, by ablation of MMP-7 in null mice, Zhou et al. [23] found renal fibrotic lesions were substantially ameliorated after UUO, which was mediated by inhibition of $\beta$-catenin activation. Overall, results from animal models indicated that MMP-7 not only was a biomarker for kidney fibrosis but also, functioned as a pathogenic mediator that promoted renal fibrosis.

Hence, we hypothesized that serum MMP-7 levels might serve as a noninvasive surrogate biomarker for disease progression in IgAN patients. Here our study verified that serum MMP-7 levels were extremely elevated in patients with IgAN. This finding differed from the result of Zhou et al, which indicated that serum MMP-7 levels were only slightly enhanced in patients with various CKD. The inconsistency might be ascribed to the different study populations. Besides, it could be also inferred that serum MMP-7 expression was elevated more significantly in IgAN, compared with other CKD.

Furthermore, our study found that serum MMP-7 levels inversely correlated with proteinuria in IgAN patients (data were not shown). These data suggested that serum MMP7 might serve as an indicator for the severity of renal dysfunction in patients with IgAN.

The Oxford classification of IgAN identified four types of items as specific pathological features which were regarded as independent predictors of renal outcome. Correlation analyses showed that serum MMP-7 levels were remarkably increased in IgAN patients exhibiting fibrotic changes, including tubulointerstitial fibrosis and glomerular sclerosis. Additionally, an overt correlation between higher serum MMP-7 level with mesangial proliferation was also noted. Taken together, these data further demonstrated that serum MMP-7 levels were positively related with kidney fibrosis in IgAN patients. 


\section{Kidney Blood Pressure Research}

From retrospective follow-up data, we found that a higher level of serum MMP-7 was independently associated with elevated risk of renal dysfunction, even in the fully adjusted model. Among cases with biopsy-proven diagnosis of IgAN, patients with the highest vs. the lowest quartile revealed a 5.173-fold greater risk of kidney disease progression. Thus, our results indicated that elevated level of serum MMP-7 may have a prognostic value in patients with IgAN. So patients with a higher level of serum MMP-7 should be warranted to receive a more intensive treatment.

Seeing the phenomenon already observed about the induction expression of MMP-7 in other fibrosis-related diseases, including diabetic nephropathy, obstructive nephropathy, and chronic allograft nephropathy [35-37], it could well be that MMP-7 is a key player in renal fibrosis, irrespective of the underlying renal disease. However, association between urine MMP-7 level and kidney disease progression was not found in type 2 diabetes, after additional adjustment for eGFR and ACR [38]. As a whole, although increase in serum MMP7 levels might be not specific for IgAN, these results still offer a potential diagnostic and prognostic values in the field of IgAN.

The strengths of our study include the relatively long follow-up, with a median of 81.9 months, which generates enough outcome events for further analyses during this period. Furthermore, adequate adjustment was performed, so that influence of confounding factors was averted as much as possible. Besides, the enzyme-linked immunosorbent assay for serum MMP-7 measurement was of a high repeatability, which provides a guarantee for the reliability of our results.

There are some limitations to this study. First, this is a retrospective follow-up study, lack of some data and imprecise is the major problem. Secondly, fibrosis degree of the renal specimens was only evaluated by Masson's trichrome staining, not Sirius red staining. Thirdly, our cohort size is rather small, and these results will need to be validated in a larger prospective cohort. Fourthly, the observed distribution of serum MMP-7 levels is distinctly skewed. To solve this problem, quartiles of serum MMP-7 levels are adopted in our regression analyses. Further, only serum samples were tested in the present study. Urine samples of larger patients will be further measured. Finally, we used a single baseline measurement of MMP-7 to predict outcome events; whether changes in MMP-7 over time add incremental predictive value is unknown.

\section{Conclusions}

In conclusion, this is the first study to demonstrate that higher serum MMP-7 level is associated with kidney fibrosis and adverse renal survival, which may serve as a noninvasive prognostic biomarker in patients with IgAN. Larger prospective cohort studies are needed to further verify its prognostic value and, whether serum MMP-7 levels vary in the course of IgAN respond to specific treatment regimens.

\section{Disclosure Statement}

No conflicts of interest exist for any authors.

\section{Acknowedgments}

This work was supported by the National Fund Committee of China (2012CB517603, 2012AA02A512, 81470938) and the Zhejiang Province Medical and Health Technology Project-2014KYA072. We appreciate the work of all colleagues and are grateful for the participation of all of the patients and healthy control subjects. 


\section{Kidney \\ Blood Pressure Research}

\section{References}

1 Wyatt RJ, Julian BA: IgA nephropathy. N Engl J Med 2013;368:2402-2414.

2 Levy M, Berger J: Worldwide perspective of IgA nephropathy. Am J Kidney Dis 1988;12:340-347.

-3 Reich HN, Troyanov S, Scholey JW, Cattran DC: Remission of proteinuria improves prognosis in IgA nephropathy. J Am Soc Nephrol 2007;18:3177-3183.

4 Moriyama T, Tanaka K, Iwasaki C, Oshima Y, Ochi A, Kataoka H, Itabashi M, Takei T, Uchida K, Nitta K: Prognosis in IgA nephropathy: 30-year analysis of 1,012 patients at a single center in Japan. PLoS One 2014;9:e91756.

5 Coppo R: Corticosteroids in IgA Nephropathy: Lessons from Recent Studies. J Am Soc Nephrol 2017;28:2533.

6 Tesar V, Troyanov S, Bellur S, Verhave JC, Cook HT, Feehally J, Roberts IS, Cattran D, Coppo R: Corticosteroids in IgA Nephropathy: A Retrospective Analysis from the VALIGA Study. J Am Soc Nephrol 2015;26:2248-2258.

7 Yoshikawa N, Honda M, Iijima K, Awazu M, Hattori S, Nakanishi K, Ito H: Steroid treatment for severe childhood IgA nephropathy: a randomized, controlled trial. Clin J Am Soc Nephrol 2006;1:511-517.

8 Berthoux FC, Mohey H, Afiani A: Natural history of primary IgA nephropathy. Semin Nephrol 2008;28:4-9.

-9 Caliskan Y, Ozluk Y, Celik D, Oztop N, Aksoy A, Ucar AS, Yazici H, Kilicaslan I, Sever MS: The Clinical Significance of Uric Acid and Complement Activation in the Progression of IgA Nephropathy. Kidney Blood Press Res 2016;41:148-157.

10 Tan M, Li W, Zou G, Zhang C, Fang J: Clinicopathological features and outcomes of IgA nephropathy with hematuria and/or minimal proteinuria. Kidney Blood Press Res 2015;40:200-206.

11 Li PK, Ho KK, Szeto CC, Yu L, Lai FM: Prognostic indicators of IgA nephropathy in the Chinese - clinical and pathological perspectives. Nephrol Dial Transplant 2002;17:64-69.

12 Cattran DC, Coppo R, Cook HT, Feehally J, Roberts IS, Troyanov S, Alpers CE, Amore A, Barratt J, Berthoux F, Bonsib S, Bruijn JA, D'Agati V, D'Amico G, Emancipator S, Emma F, Ferrario F, Fervenza FC, Florquin S, Fogo A, Geddes CC, Groene HJ, Haas M, Herzenberg AM, Hill PA, Hogg RJ, Hsu SI, Jennette JC, Joh K, Julian BA, Kawamura T, Lai FM, Leung CB, Li LS, Li PK, Liu ZH, Mackinnon B, Mezzano S, Schena FP, Tomino Y, Walker PD, Wang H, Weening JJ, Yoshikawa N, Zhang H: The Oxford classification of IgA nephropathy: rationale, clinicopathological correlations, and classification. Kidney Int 2009;76:534-545.

-13 Coppo R, Troyanov S, Bellur S, Cattran D, Cook HT, Feehally J, Roberts IS, Morando L, Camilla R, Tesar V, Lunberg S, Gesualdo L, Emma F, Rollino C, Amore A, Praga M, Feriozzi S, Segoloni G, Pani A, Cancarini G, Durlik M, Moggia E, Mazzucco G, Giannakakis C, Honsova E, Sundelin BB, Di Palma AM, Ferrario F, Gutierrez E, Asunis AM, Barratt J, Tardanico R, Perkowska-Ptasinska A: Validation of the Oxford classification of IgA nephropathy in cohorts with different presentations and treatments. Kidney Int 2014;86:828-836.

14 Alamartine E, Sauron C, Laurent B, Sury A, Seffert A, Mariat C: The use of the Oxford classification of IgA nephropathy to predict renal survival. Clin J Am Soc Nephrol 2011;6:2384-2388.

15 Tanaka S, Ninomiya T, Katafuchi R, Masutani K, Tsuchimoto A, Noguchi H, Hirakata H, Tsuruya K, Kitazono T: Development and validation of a prediction rule using the Oxford classification in IgA nephropathy. Clin J Am Soc Nephrol 2013;8:2082-2090.

16 Jiang H, Liang L, Qin J, Lu Y, Li B, Wang Y, Lin C, Zhou Q, Feng S, Yip SH, Xu F, Lai EY, Wang J, Chen J: Functional networks of aging markers in the glomeruli of IgA nephropathy: a new therapeutic opportunity. Oncotarget 2016;7:33616-33626.

17 Tan RJ, Liu Y: Matrix metalloproteinases in kidney homeostasis and diseases. Am J Physiol Renal Physiol 2012;302:F1351-1361.

18 Ahmed AK, El Nahas AM, Johnson TS: Changes in matrix metalloproteinases and their inhibitors in kidney transplant recipients. Exp Clin Transplant 2012;10:332-343.

19 McGuire JK, Li Q, Parks WC: Matrilysin (matrix metalloproteinase-7) mediates E-cadherin ectodomain shedding in injured lung epithelium. Am J Pathol 2003;162:1831-1843.

20 Rodder S, Scherer A, Korner M, Marti HP: A subset of metzincins and related genes constitutes a marker of human solid organ fibrosis. Virchows Arch 2011;458:487-496. 


\section{Kidney \\ Blood Pressure Research}

-21 White ES, Xia M, Murray S, Dyal R, Flaherty CM, Flaherty KR, Moore BB, Cheng L, Doyle TJ, Villalba J, Dellaripa PF, Rosas IO, Kurtis JD, Martinez FJ: Plasma Surfactant Protein-D, Matrix Metalloproteinase-7, and Osteopontin Index Distinguishes Idiopathic Pulmonary Fibrosis From Other Idiopathic Interstitial Pneumonias. Am J Respir Crit Care Med 2016;194:1242-1251.

22 Huang CC, Chuang JH, Chou MH, Wu CL, Chen CM, Wang CC, Chen YS, Chen CL, Tai MH: Matrilysin (MMP-7) is a major matrix metalloproteinase upregulated in biliary atresia-associated liver fibrosis. Mod Pathol 2005;18:941-950.

-23 Zhou D, Tian Y, Sun L, Zhou L, Xiao L, Tan RJ, Tian J, Fu H, Hou FF, Liu Y: Matrix Metalloproteinase-7 Is a Urinary Biomarker and Pathogenic Mediator of Kidney Fibrosis. J Am Soc Nephrol 2017;28:598-611.

-24 He W, Tan RJ, Li Y, Wang D, Nie J, Hou FF, Liu Y: Matrix metalloproteinase-7 as a surrogate marker predicts renal Wnt/beta-catenin activity in CKD. J Am Soc Nephrol 2012;23:294-304.

-25 Hennino MF, Buob D, Van der Hauwaert C, Gnemmi V, Jomaa Z, Pottier N, Savary G, Drumez E, Noel C, Cauffiez C, Glowacki F: miR-21-5p renal expression is associated with fibrosis and renal survival in patients with IgA nephropathy. Sci Rep 2016;6:27209.

-26 Barbour SJ, Espino-Hernandez G, Reich HN, Coppo R, Roberts IS, Feehally J, Herzenberg AM, Cattran DC: The MEST score provides earlier risk prediction in IgA nephropathy. Kidney Int 2015, DOI 10.1038/ ki.2015.322.

27 Liu X, Wang Y, Wang C, Shi C, Cheng C, Chen J, Ma H, Lv L, Li L, Lou T: A new equation to estimate glomerular filtration rate in Chinese elderly population. PLoS One 2013;8:e79675.

28 Levey AS, Stevens LA, Schmid CH, Zhang YL, Castro AF, 3rd, Feldman HI, Kusek JW, Eggers P, Van Lente F, Greene T, Coresh J: A new equation to estimate glomerular filtration rate. Ann Intern Med 2009;150:604612.

-29 Haas M, Verhave JC, Liu ZH, Alpers CE, Barratt J, Becker JU, Cattran D, Cook HT, Coppo R, Feehally J, Pani A, Perkowska-Ptasinska A, Roberts IS, Soares MF, Trimarchi H, Wang S, Yuzawa Y, Zhang H, Troyanov S, Katafuchi R: A Multicenter Study of the Predictive Value of Crescents in IgA Nephropathy. J Am Soc Nephrol 2017;28:691-701.

-30 Donadio JV, Grande JP: IgA nephropathy. N Engl J Med 2002;347:738-748.

-31 Genovese F, Boor P, Papasotiriou M, Leeming DJ, Karsdal MA, Floege J: Turnover of type III collagen reflects disease severity and is associated with progression and microinflammation in patients with IgA nephropathy. Nephrol Dial Transplant 2016;31:472-479.

-32 Glowacki F, Savary G, Gnemmi V, Buob D, Van der Hauwaert C, Lo-Guidice JM, Bouye S, Hazzan M, Pottier N, Perrais M, Aubert S, Cauffiez C: Increased circulating miR-21 levels are associated with kidney fibrosis. PLoS One 2013;8:e58014.

-33 Fang Y, Yu X, Liu Y, Kriegel AJ, Heng Y, Xu X, Liang M, Ding X: miR-29c is downregulated in renal interstitial fibrosis in humans and rats and restored by HIF-alpha activation. Am J Physiol Renal Physiol 2013;304:F1274-1282.

34 Szeto CC, Ching-Ha KB, Ka-Bik L, Mac-Moune LF, Cheung-Lung CP, Gang W, Kai-Ming C, Kam-Tao LP: Micro-RNA expression in the urinary sediment of patients with chronic kidney diseases. Dis Markers 2012;33:137-144.

-35 Surendran K, Simon TC, Liapis H, McGuire JK: Matrilysin (MMP-7) expression in renal tubular damage: association with Wnt4. Kidney Int 2004;65:2212-2222.

-36 Ban CR, Twigg SM, Franjic B, Brooks BA, Celermajer D, Yue DK, McLennan SV: Serum MMP-7 is increased in diabetic renal disease and diabetic diastolic dysfunction. Diabetes Res Clin Pract 2010;87:335-341.

-37 Ho J, Rush DN, Krokhin O, Antonovici M, Gao A, Bestland J, Wiebe C, Hiebert B, Rigatto C, Gibson IW, Wilkins JA, Nickerson PW: Elevated Urinary Matrix Metalloproteinase-7 Detects Underlying Renal Allograft Inflammation and Injury. Transplantation 2016;100:648-654.

-38 Afkarian M, Zelnick LR, Ruzinski J, Kestenbaum B, Himmelfarb J, de Boer IH, Mehrotra R: Urine matrix metalloproteinase-7 and risk of kidney disease progression and mortality in type 2 diabetes. J Diabetes Complications 2015;29:1024-1031. 